\title{
FR-167653, a selective p38 MAPK inhibitor, exerts salutary effect on liver cirrhosis through downregulation of Runx2
}

\author{
Shinji Hattori ${ }^{1}$, Dipok K Dhar ${ }^{2}$, Nobumasa Hara ${ }^{3}$, Yasuhito Tonomoto ${ }^{1}$, Toshinao Onoda ${ }^{1}$, Takashi Ono $^{1}$, \\ Akira Yamanoi ${ }^{1}$, Mitsuo Tachibana ${ }^{1}$, Mikako Tsuchiya ${ }^{3}$ and Naofumi Nagasue ${ }^{1}$
}

Liver cirrhosis remains a difficult-to-treat disease with a substantial morbidity and mortality rate. There is an emerging body of data purporting a pivotal role of the activated p38 mitogen-activated protein kinase (MAPK) in the process of cirrhosis. Several anticirrhotic agents have been developed over the past few years, and most of them exert their effects by indirectly inhibiting the p38 pathway. Effect of a selective p38 inhibitor is yet to be reported. In this study, we evaluated the salutary effect of FR-167653 (FR), a selective p38 inhibitor, in a carbon tetrachloride $\left(\mathrm{CCl}_{4}\right)$-induced rat cirrhotic model. Twenty rats were assigned into four groups: Sham, olive oil only; Control, $\mathrm{CCl}_{4}$ in olive oil; FR50, FR $50 \mathrm{mg} /$ $\mathrm{kg} /$ day and $\mathrm{CCl}_{4}$; and $\mathrm{FR} 100, \mathrm{FR} 100 \mathrm{mg} / \mathrm{kg} /$ day and $\mathrm{CCl}_{4}$. FR dose-dependently inhibited activation of $\mathrm{p} 38$ and had an ameliorating effect on cirrhosis formation. Significant dose-dependent reduction in $\alpha$-smooth muscle actin immunostaining and hydroxyproline content of the liver was noticed in the FR-treated rats. Also densitometric analysis showed a significant reduction in azan-stained area in the FR-treated rats. These fibrotic changes were observed in the myofibroblasts including the hepatic stellate cells and portal fibroblasts. mRNA expression of runt-related protein 2 (Runx2), a profibrogenic transcription factor, was significantly low in FR-treated livers, indicating that Runx2 might be a key downstream regulator of the p38 pathway. A similar reduction in expression of Smad4 and tissue inhibitor of metalloproteinase-1 was noticed in the FR-treated rats. In conclusion, FR treatment exerted a significant beneficial effect in a $\mathrm{CCl}_{4}$-induced rat cirrhotic model. The ameliorating effect of $\mathrm{FR}$ could be partially attributable to an inhibition of the Smad4/p38/Runx2 axis in the cirrhotic liver.

Laboratory Investigation (2007) 87, 591-601; doi:10.1038/labinvest.3700539; published online 5 March 2007

KEYWORDS: fibrosis; hepatic stellate cell; p38 MAPK; Runx2; Smad; TGF- $\beta$

Liver cirrhosis is characterized by an awry scarring process in response to chronic or iterative hepatocyte injury. This scarring process is the body's natural response to noxious injuries to hepatocytes, and represents a paradigm for wound healing in other sites. Exaggerated fibrotic process in the liver mainly involves the perisinusoidal spaces and thus produces detrimental effect on the splanchnic blood flow resulting in portal hypertension. To date, it is understood that hepatic stellate cells (HSCs) comprise $15 \%$ of the total number of resident liver cells, are the most important collagen-producing cells during the development of the hepatic fibrosis. However, several recent reports showed that in addition to the HSCs, portal fibroblasts, second-layer cells surrounding the centrilobular veins, fibroblasts present in the Glisson capsule and vascular smooth muscle cells may also express myofiroblastic phenotype and are involved in fibrogenesis. ${ }^{1-4}$ Following noxious injury to the hepatocytes, inflammatory cells infiltrate into the liver and these activated mesenchymal cells produce extracellular matrix (ECM), cytokines and chemotactic factors, and activate the fibrogenic process. Although the pathological process of cirrhosis is well recognized, the intracellular signaling pathways controlling collagen formation by the activated myofibroblasts are not yet clearly understood.

\footnotetext{
${ }^{1}$ Department of Digestive and General Surgery, Faculty of Medicine, Shimane University, Izumo, Japan; ${ }^{2}$ Department of Medicine, Institute of Hepatology, University College of London, London, UK and ${ }^{3}$ Department of Biochemistry, Faculty of Medicine, Shimane University, Izumo, Japan 
It is assumed that a concerted activity between multiple cytokines and growth factors including the transforming growth factor- $\beta$ (TGF- $\beta),{ }^{5}$ activinA,${ }^{6}$ endothelin, ${ }^{7}$ plateletderived growth factor (PDGF), ${ }^{8}$ epidermal growth factor $(\mathrm{EGF}),{ }^{9}$ and tumor necrosis factor- $\alpha(\mathrm{TNF}-\alpha)^{10}$ is essential for increased fibrogenesis. Nevertheless, an altered balance between the matrix-degrading enzymes (MMPs; matrix metalloproteinases) and their inhibitors (TIMPs; tissue inhibitors of matrix protease) is crucial for the deposition of collagen fibers. In cirrhotic livers, activated HSCs produce an excess amount of TIMP-1 and prevent matrix degradation. ${ }^{11}$ Accumulated evidence shows that several of these profibrogenic factors were used as a target to design effective therapy for cirrhosis in animal models. However, the ideal one is yet to be detected for use in the clinic. It is worth mentioning that most of these antifibrotic agents exert their effect through a common signaling pathway, which converges at p38 mitogen-activated protein kinase (MAPK)-mediated activation of myofibroblasts. Therefore, it seems that inhibition of p38 MAPK pathway may prove effective in treating liver cirrhosis.

Members of the MAPK family represent a superfamily of serine/threonine kinases that play a major role in the signaling pathway linking diverse extracellular signals such as oxidative stress, osmotic stress, heat shock, ultraviolet irradiation, ischemia-reperfusion, lipopolysaccaride, inflammatory cytokines, and DNA-damaging agents to vital cellular processes. The MAPK superfamily includes the extracellular signal-regulated kinases (ERK1/ERK2/5), c-Jun $\mathrm{N}$-terminal kinases (JNKs), and p38 MAPK. ${ }^{12}$ Each of these MAPK is activated by phosphorylation and subsequently translocates into the cell nucleus. In turn, they regulate transcription of specific genes, which are involved in either cellular apoptosis or survival depending on the context, inflammatory response and tissue remodeling. Results of several studies show that among the members of the MAPK, the p38 MAPK signaling pathway is specifically involved in the activation of myofibroblasts by most of the liver cirrhosis-inducing agents including TGF- $\beta{ }^{13}$

The runt-related protein (Runx) family of transcription factors encoded by three distinct genes, Runx1, Runx2, and Runx3, plays pivotal roles during normal development and in neoplasia. ${ }^{14}$ Among the three members of the Runx family, Runx2 plays an essential role in osteoblast differentiation and bone formation. Recently, it has been reported that Runx2 is a new important downstream target of the TGF/Smad4 pathway, and p38 plays an important role in signal transduction between Smad4 and Runx2. During osteogenesis, Runx2 regulates collagen $1-\alpha$ expression by binding the osteoblast-specific cis-acting elements (OSE2). ${ }^{15}$ Also MAPK pathway plays an essential role in phosphorylation of Runx2. ${ }^{16}$ However, the exact role of Runx2 in cirrhosis formation and its relationship with modulation of the p38 MAPK pathway during cirrhosis are yet to be reported.
FR-167653 (FR), a small molecule p38 MAPK inhibitor, which suppresses the production of TNF- $\alpha$ and interleukin$1 \beta$ (IL-1 $\beta)$, in a variety of experimental settings has been reported. To date, several studies have evaluated its beneficial effect in animal models of disseminated intravascular coagulation, endotoxin-induced shock, Helicobacter pylori-induced gastritis, glomerulonephritis, ${ }^{17}$ pulmonary fibrosis, ${ }^{18}$ hepatic ischemia-reperfusion injury ${ }^{19}$ and acute hepatic injury. ${ }^{20}$ However, the role of FR in liver cirrhosis still remains elusive.

To that end, in this study, we sought to determine the antifibrotic effect of FR in a carbon tetrachloride- $\left(\mathrm{CCl}_{4}-\right)$ induced rat cirrhotic model. Furthermore, we also found that Runx2 might be one of the downstream targets of the p38 MAPK pathway and the ameliorating effect of FR on liver cirrhosis could be partially attributable to the downregulation of Runx2 in rat livers.

\section{MATERIALS AND METHODS \\ Animals}

Male Sprague-Dawley rats, 6 weeks old and weighing around $180 \mathrm{~g}$, were obtained from CLEA Japan Inc. (Tokyo, Japan). The animals were housed in an air-conditioned room maintained at $23 \pm 2^{\circ} \mathrm{C}$, with a relative humidity of $50 \pm 10 \%$ and alternate light and dark conditions. They were given standard laboratory rodent chow (Oriental Yeast, Tokyo). Animals received humane care in accordance with the guidelines of the department of experimental animals for integrated research in science, Shimane University.

\section{Experimental Models}

As previously described, liver cirrhosis was induced, by subcutaneous injection of $50 \% \mathrm{CCl}_{4}$ at a dose of $2 \mathrm{ml} / \mathrm{kg}$ twice a week along with phenobarbital in drinking water $(800 \mathrm{mg} / \mathrm{l})$. FR-167653 (FR) was a generous gift from the Fujisawa Pharmaceutical Co. Ltd. (Osaka, Japan) and was dissolved in polyethylene glycol (PEG) for use in rats. Rats were assigned randomly into one of the four groups: (1) Sham group $(n=5)$-rats were injected subcutaneously with olive oil only $(250 \mu \mathrm{l})$, (2) Controls $(n=5)$-vehicle only (250 $\mu \mathrm{l} \mathrm{PEG),} \mathrm{(3)} \mathrm{FR50} \mathrm{treatment} \mathrm{group} \mathrm{(} n=5$ ) -rats were injected subcutaneously with 5\% FR in a volume of $250 \mu \mathrm{l}$ PEG, and (4) FR100 treatment group $(n=5)$-rats were injected subcutaneously with $10 \%$ FR in a volume of $250 \mu \mathrm{l}$ PEG. Body weight of all animals was monitored regularly. After treating for a 1-month period, all animals were humanely killed. Immediately before killing, blood samples were collected from the abdominal vena cava for measurement of biochemical markers of hepatic function (albumin, AST, ALT, $\gamma$ GTP). Livers were carefully dissected and one portion was immediately frozen in liquid nitrogen for preservation and another portion was fixed in paraformaldehyde to prepare paraffin-embedded tissue sections for staining purposes. 


\section{Measurement of Hepatic Hydroxyproline Content}

For measurement of hepatic hydroxyproline levels, a portion of liver tissue was homogenized in 0.25 volumes $(\mathrm{ml} / \mathrm{mg})$ of $6 \mathrm{~N} \mathrm{HCl}$ at $4^{\circ} \mathrm{C}$ with a Polytron homogenizer. Homogenized liver specimens were hydrolyzed at $110^{\circ} \mathrm{C}$ in an autoclave at a pressure of $1.2 \mathrm{kgf} / \mathrm{cm}^{2}$ for $24 \mathrm{~h}$. After centrifugation at 2000 r.p.m. at $4^{\circ} \mathrm{C}$ for $5 \mathrm{~min}$, the supernatant was used for quantitative determination. Quantitative determination of hepatic hydroxyproline levels was performed using a highperformance liquid chromatographic (HPLC) system (SRL Inc., Tachikawa, Japan). All samples were measured in duplicate.

\section{Histopathology}

Formalin-fixed paraffin-embedded liver sections $(5 \mu \mathrm{m})$ were used for $\mathrm{H} \& \mathrm{E}$ and azan staining. For semiquantitative analysis of cirrhosis, the most extensively stained area of the liver section was identified under low magnification $(\times 40)$ and later azan-stained area was measured at higher magnification in at least five high power fields $(\times 100)$. Digital images were captured by using a charge-coupled device video camera (HC-300/OL; OLYMPUS, Tokyo, Japan) and a color image freezer computer software (Photograb-300 SH-3; FUJIFILM, Tokyo, Japan), and stored on optical disks. Quantification of the azan-stained area was carried out by a public domain $\mathrm{NIH}$ image program (developed at the US National Instituted of Health and available on the internet at http://rsb.info. nih.gov/nih-image/) on a Macintosh computer. Low frequency background was removed with the 'background subtraction' tool and azan-stained area was measured as number of pixels per unit area of liver section (2.8 million pixels).

\section{Immunohistochemistry}

Paraffin-embedded sections were deparaffinized in xylene and rehydrated in graded ethanols. The sections were boiled in $10 \mathrm{mM}$ sodium citrate ( $\mathrm{pH} 6.0$ ) for $10 \mathrm{~min}$ and incubated in $3 \% \mathrm{H}_{2} \mathrm{O}_{2}$ for $20 \mathrm{~min}$. The slides were then washed with PBS three times for $5 \mathrm{~min}$. After treatment with blocking serum for $30 \mathrm{~min}$, the sections were incubated with the primary antibodies (1) monoclonal mouse IgG against $\alpha$-smooth muscle actin (SMA) (Dako Inc., Kyoto, Japan) at 1:50 dilution overnight, (2) monoclonal mouse IgG against Smad4 (Santa Cruz Biotechnology Inc., Santa Cruz, CA, USA) at 1:300 dilution overnight, and (3) monoclonal mouse IgG against TIMP-1 (Daiichi Fine Chemical Inc., Toyama, Japan) at 1:40 dilution overnight. The immunohistochemical staining was carried using the streptoavidin-biotin kit (Nichirei, Japan) by the avidin-biotin-peroxidase method, according to the manufacturer's instructions. Aminoethylcalbasol was used as the chromogen. Formalin-fixed, paraffin-embedded sections of human and mouse colon tissue were used as positive controls for $\alpha$-SMA and Smad4; sections of human breast tissue were used as positive controls for TIMP-1. Sections without primary antibodies as well as those with nonimmunized mouse serum IgG (Daiichi Fine Chemical Inc., Toyama, Japan) served as negative controls. Semiquantitative analysis of $\alpha$-SMA-stained area was carried out with an image analyzer as described above for azan staining. Staining of Smad4 was observed mostly in the nucleus of cells. The number of Smad4 positive nucleus was counted in at least 20 high-power fields. Nuclear immunoreactivity for Smad4 was described as the percentage of the number of positive myofibroblasts in 1000 myofibroblasts that were spindle shaped.

\section{Western Blot Analysis}

Liver tissues were lysed in an extraction buffer containing $50 \mathrm{mmol} / \mathrm{l}$ of Tris-hydrochloric acid ( $\mathrm{pH} \mathrm{7.5),} 150 \mathrm{mmol} / \mathrm{l}$ of sodium chloride, $1 \mathrm{mmol} / \mathrm{l}$ of sodium orthovanadate, $1 \mathrm{mmol} / \mathrm{ml}$ of ethylenediaminetetraacetic acid (EDTA), $1 \mathrm{mmol} / \mathrm{ml}$ of ethyleneglycotetraacetic acid, and $1 \mathrm{mmol} / \mathrm{l}$ of phenylmethylsulfonyl fluoride. Samples were homogenized in $1 \mathrm{ml}$ of extraction buffer on ice. All debris and nuclei were removed by centrifugation at 15000 r.p.m. at $4{ }^{\circ} \mathrm{C}$ for $10 \mathrm{~min}$, and the supernatant obtained was used for Western blot analysis. Total protein content was determined by the Protein Assay CBB solution (Nacali tesque, Kyoto, Japan) with bovine serum albumin as the reference standard. Fifty micrograms of protein for each sample were electrophoresed on sodium dodecyl sulfate-polyacrylamide gels, and transferred to polyvinylidene difluoride (PVDF) membrane. The PVDF membranes were incubated with 5\% skim milk in 0.05\% Tween-20-Tris-buffered saline (TTBS) and washed three times in $0.05 \%$ TTBS. The membranes were treated with primary antibodies (1) polyclonal rabbit IgG against p38 MAPK (Santa Cruz Biotechnology Inc., Santa Cruz, CA, USA) at 1:5000 dilution for $2 \mathrm{~h}$ at room temperature and (2) polyclonal rabbit IgG against phosphorylated p38 MAPK (Cell Signaling, Beverly, MA, USA) at 1:1000 dilution overnight at $4{ }^{\circ} \mathrm{C}$. After three washings in $0.05 \%$ TTBS, the membranes were incubated with goat anti-rabbit IgG horseradish peroxidase-conjugated secondary antibody (antip38 MAPK and anti-phospho p38 MAPK; 1:8000 and 1:18000 dilution for $1 \mathrm{~h}$ at room temperature). After being washed three times in $0.05 \%$ TTBS, the membranes were incubated with the ECL reagent (Amersham International PLC, UK) for $30 \mathrm{~s}$ and exposed to X-ray film. Lysates of HEK cells with and without an anisomycin and ultraviolet irradiation served as positive controls. For evaluation of the activity of p38 MAPK, ratio of phosphorylated p38 MAPK and total p38 MAPK were analyzed by densitometric analysis with the NIH image analyzer.

\section{RT-PCR}

Total RNA was isolated according to a single-step method with TRI REAGENT (Molecular Research Center Inc., Montgomery). First-strand cDNA was synthesized from $5 \mu \mathrm{g}$ of total RNA using Super Script II first-strand synthesis system with oligo $(\mathrm{dT})_{13}$ as per the manufacturer's instruc- 
tions (Invitrogen, Carlsbad, CA, USA). The cDNA samples were amplified using a thermal cycler (Program Temp. Control System PC-701, ASTEC, Fukuoka, Japan) with the following sets of primers. For Runx-2, $5^{\prime}$-ctgagccagatgacgtcc$3^{\prime}$ (sense primer), $5^{\prime}$-tggctttgggaagagccg- $3^{\prime}$ (antisense primer); for $\beta$-actin, $5^{\prime}$-agagggaaatcgtgcgtgaca- $3^{\prime}$ (sense primer), $5^{\prime}$-acatctgctggaaggtggaca- $3^{\prime}$ (antisense primer). These primers for each gene were derived from different exons, and the sequences of the amplified products were confirmed to ensure that they represent the specific mRNA species and not genomic DNA. Negative controls (cDNA-free solutions) were included in each run. Each cDNA species was amplified for 31 cycles (Runx2) and 18 cycles ( $\beta$-actin) $\left(94^{\circ} \mathrm{C}\right.$ for $35 \mathrm{~s}, 55^{\circ} \mathrm{C}$ for $35 \mathrm{~s}$, and $72^{\circ} \mathrm{C}$ for $2 \mathrm{~min}$ ), followed by a final extension at $72^{\circ} \mathrm{C}$ for $4 \mathrm{~min}$. After defined cycles of PCR, from the $25 \mu \mathrm{l}$ reaction volume, $9 \mu \mathrm{l}$ was electrophoresed on $1.5 \%$ agarose gel in $0.5 \mathrm{M}$ Tris-borate-EDTA buffer, and amplified bands were detected by ethidium bromide staining. The intensity of ethidium bromide fluorescence was measured by a charged couple device system (Kodak Digital Science EDAS290 System, Invitrogen Japan K.K., Japan). For quantification of Runx2 mRNA, the samples were evaluated in comparison with the PCR product to $\beta$-actin.

\section{Biochemical Assay of the Serum Samples}

The serum levels of albumin, AST, ALT, $\gamma$ GTP were determined by an automated analyzer (TBA-200FR, Toshiba Inc., Japan) as per the manufacturer's instructions.

\section{Statistical Analysis}

Statistical analysis was performed using the Statview J-5.0 program. Numerical data represent mean \pm s.d. of individual experiments unless otherwise stated. Statistical difference was determined by Student's $t$-test. $P<0.05$ indicated a statistically significant difference.

\section{RESULTS}

\section{Body Weight Gain While Killing}

FR50 rats had a significant increase in body weight when compared with the $\mathrm{CCl}_{4}$ rats $\left(\mathrm{CCl}_{4}\right.$ vs $\mathrm{FR} 50$; mean \pm s.d., $103.0 \pm 14.0$ vs $129.0 \pm 8.9 \mathrm{~g} ; P<0.01)$. However, a similar weight gain was not detected in FR100 rats $(93.0 \pm 6.7 \mathrm{~g})$ (Table 1). Indeed, rats in the FR100 group had anorexia, bleeding tendencies, and bruises, as side effects, in the later half of treatment period and thus the lower body weight could be attributable to these side effects of high dose of FR $(100 \mathrm{mg} / \mathrm{kg} /$ day $)$ in rats.

\section{Effect on Serum Biochemical Markers}

Serum levels of AST and ALT in the FR-treated rats (50, $100 \mathrm{mg} / \mathrm{kg} /$ day) were significantly lower than those in the $\mathrm{CCl}_{4}$ group, indicating that $\mathrm{FR}$ inhibited the hepatotoxic effect of $\mathrm{CCl}_{4}$ and thus improved liver function. However, the serum level of albumin in the FR100 group was significantly lower than those in the $\mathrm{CCl}_{4}$ and FR50 group, indicating a
Table 1 Body weight gain and serum biochemical maker

\begin{tabular}{lcccc}
\hline Groups & Olive oil $(n=5)$ & $\mathrm{CC}_{14}(n=5)$ & FR50 $(n=5)$ & FR100 $(n=5)$ \\
\hline BW gain (g) & $158.2 \pm 22.7$ & $103.0 \pm 14.0^{\mathrm{a}}$ & $129.0 \pm 8.9^{\mathrm{b}, \mathrm{c}}$ & $93.0 \pm 6.7^{\mathrm{a}, \mathrm{e}}$ \\
Alb (g/dl) & $3.92 \pm 0.31$ & $3.84 \pm 0.29$ & $3.72 \pm 0.19$ & $3.14 \pm 0.25^{\mathrm{a}, \mathrm{c}, \mathrm{e}}$ \\
AST (IU/l) & $76.2 \pm 4.0$ & $161.2 \pm 63.0^{\mathrm{b}}$ & $86.8 \pm 24.1^{\mathrm{d}}$ & $75.8 \pm 13.7^{\mathrm{d}}$ \\
ALT (IU/l) & $37.0 \pm 4.7$ & $126.0 \pm 46.0^{\mathrm{a}}$ & $64.6 \pm 23.8^{\mathrm{b}, \mathrm{d}}$ & $45.2 \pm 16.2^{\mathrm{c}}$ \\
$\gamma$-GTP (IU/I) & $0.6 \pm 0.5$ & $22.2 \pm 19.0^{\mathrm{b}}$ & $5.0 \pm 4.2^{\mathrm{b}}$ & $5.2 \pm 1.1^{\mathrm{a}}$ \\
\hline
\end{tabular}

Note: The results were expressed as means \pm s.d. Olive oil, olive oil alone; $\mathrm{CCl}_{4}$, $\mathrm{CCl}_{4}$ alone. $\mathrm{FR} 50, \mathrm{CCl}_{4}$ with $\mathrm{FR} 16765350 \mathrm{mg} / \mathrm{kg} /$ day. $\mathrm{FR} 100, \mathrm{CCl}_{4}$ with FR167653 $100 \mathrm{mg} / \mathrm{kg} /$ day.

${ }^{\mathrm{a}} P<0.01$ vs olive oil.

${ }^{\mathrm{b}} P<0.05$ vs olive oil.

${ }^{\mathrm{c}} P<0.01$ vs $\mathrm{CCl}_{4}$.

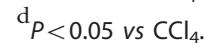

$\mathrm{e}_{P<0.01 \text { vs FR50. }}$

poor nutritional status and might be attributable to the anorexic effect of high dose of FR in this model of $\mathrm{CCl}_{4}$ induced cirrhotic rats (Table 1).

\section{Histopathology, Azan and $\alpha$-SMA Staining}

Microscopic analysis of liver sections showed typical cirrhotic changes in the $\mathrm{CCl}_{4}$ rats with distortion of hepatic lobules, formation of thick fibrous septa, frequent infiltration of inflammatory cells, and nodular regeneration of hepatocytes (Figure 1). The fibrotic changes were predominantly noticed as portal-central bridges and, also in the periportal, pericentral, and in perisinusoidal areas. In contrast to $\mathrm{CCl}_{4}$, FRtreatment dramatically reduced azan-stained area and only traces of staining could be noticed surrounding the portal and central veins. In FR-treated rats, the liver structure was well preserved and inflammatory cells were sparse.

Densitometric analysis of azan-stained area showed that the azan-stained fibrotic area was significantly higher in $\mathrm{CCl}_{4}$ rats when compared with the FR-treated rats $\left(\mathrm{CCl}_{4} v s\right.$ FR50; $23915.5 \pm 7257.9$ vs $9746.0 \pm 4029.5 ; P<0.01$ and $\mathrm{CCl}_{4}$ vs FR100; $23915.5 \pm 7257.9$ vs $\quad 6060.8 \pm 1624.5 ; \quad P<0.01)$ (Figure 1).

As it was noticed for azan staining, the $\alpha$-SMA-stained area was also sparse in both groups of FR-treated rats, than in the $\mathrm{CCl}_{4}$ rats. In the $\mathrm{CCl}_{4}$ rats, $\alpha$-SMA was strongly expressed in the space of Disse where HSCs were located, and in the portal-central bridges. In contrast to $\mathrm{CCl}_{4}$, FR-treatment caused a significant reduction in $\alpha$-SMA-stained area, and the effect was noticed predominantly in the perisinusoidal area and reduction was somewhat weaker periportally. Densitometric assessment of the $\alpha$-SMA-stained area showed a significant dose-dependent reduction in the $\alpha$-SMA-stained area in both FR50 and FR100 groups when compared with the $\mathrm{CCl}_{4}\left(\mathrm{CCl}_{4}\right.$ vs $\mathrm{FR} 50 ; 64605.1 \pm 9070.9$ vs $33506.9 \pm 13087.8$; 

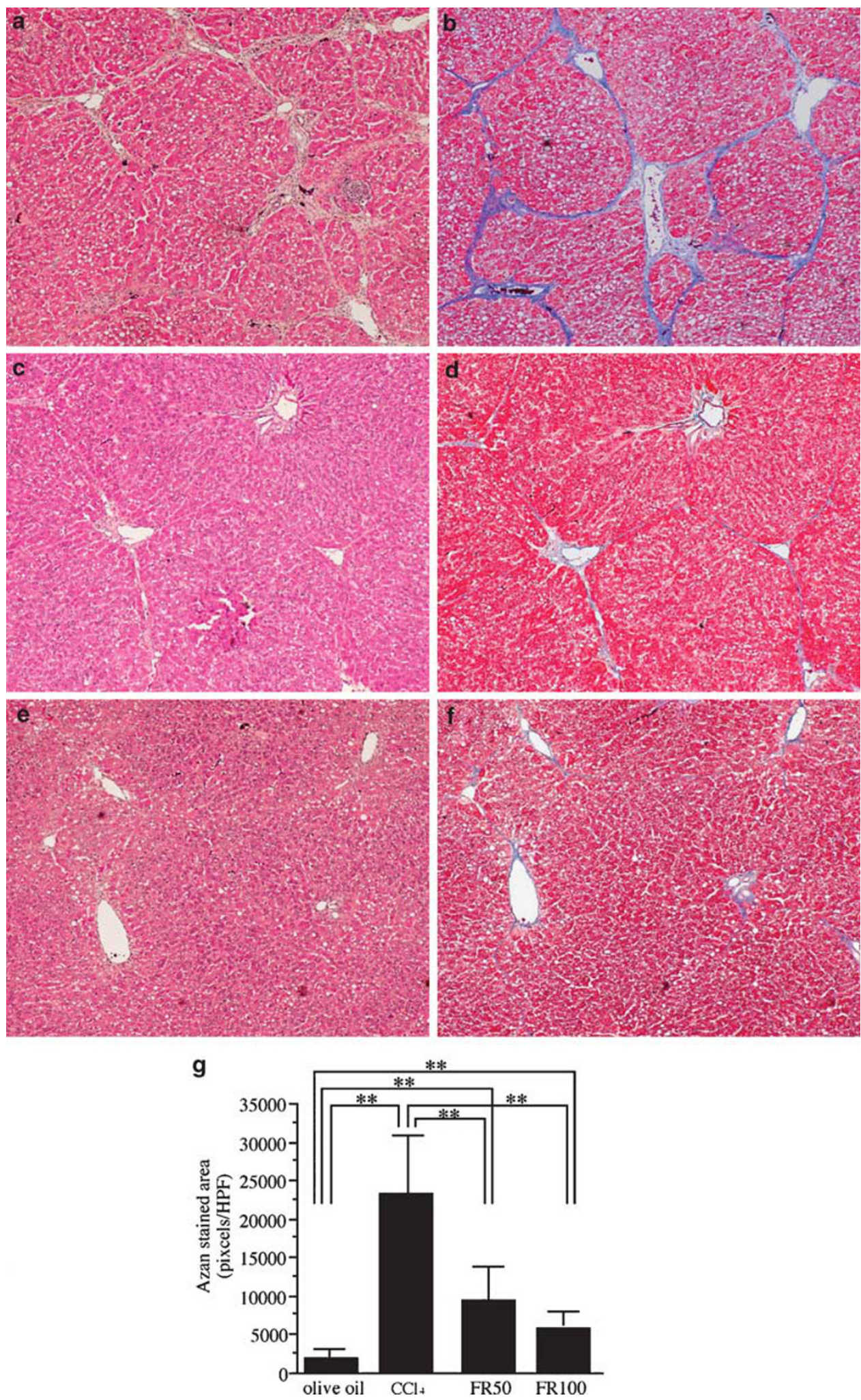

Figure 1 Histopathological sections of representative liver sections showing $\mathrm{H} \& \mathrm{E}$ and azan-stained areas. (a and $\mathbf{b}) \mathrm{CCl}_{4}$, (c and d) $\mathrm{FR} 50 \mathrm{mg} / \mathrm{kg} / \mathrm{day}$, (e and f) FR $100 \mathrm{mg} / \mathrm{kg} /$ day (a, c, and $\mathbf{e} ; \mathrm{H}$ and E staining, b, d, and f; azan staining, $\times 100)$. (g) Semiquantitative analyses of azan-stained areas showed a significant reduction of azan-stained area in both FR50 and FR100 than in $\mathrm{CCl}_{4}$ rats. Results are expressed as mean \pm s.d. ${ }^{\star} P<0.05,{ }^{* * P}<0.01$. 

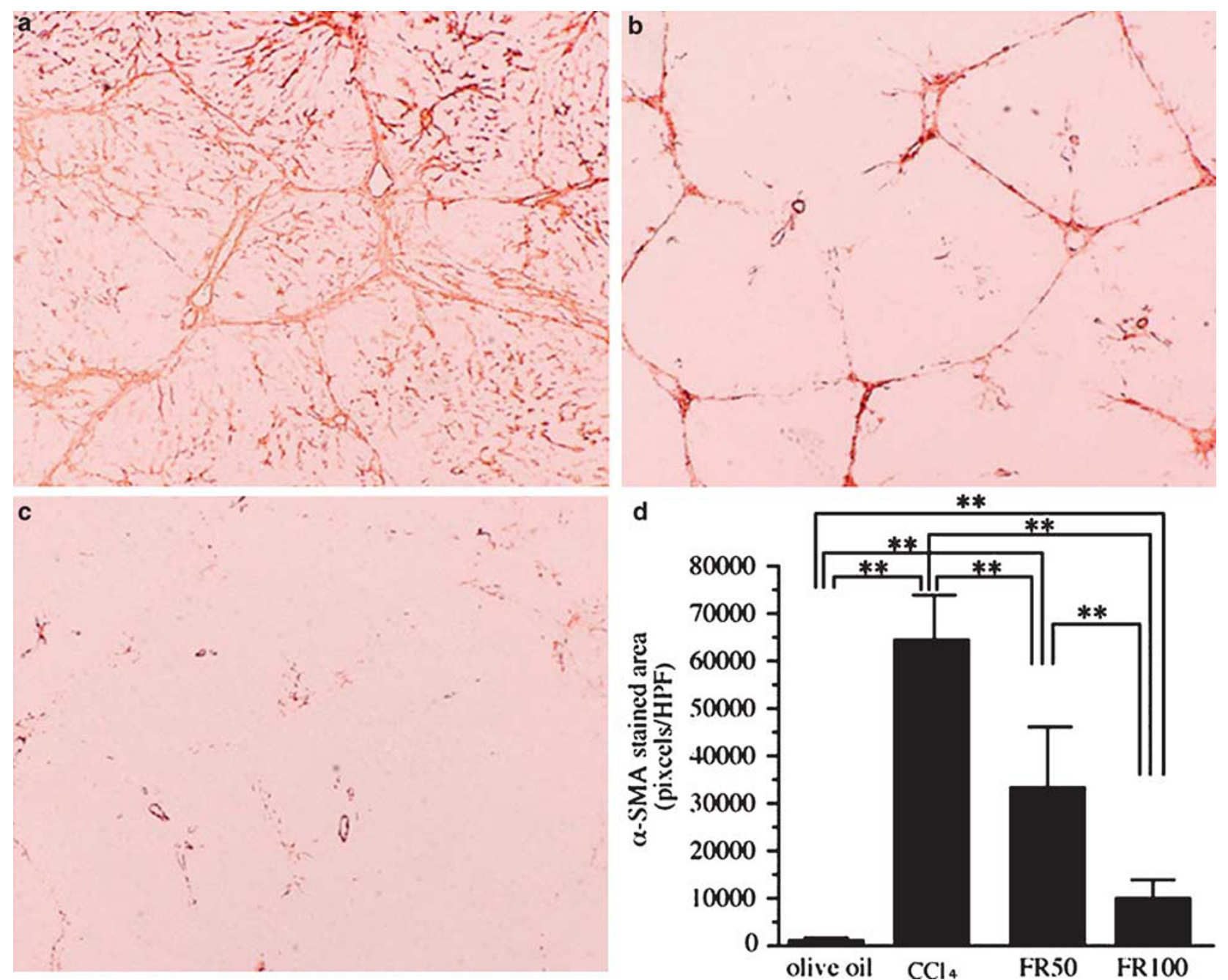

Figure 2 Immunohistochemistry of $\alpha$-SMA in representative liver sections showing $\alpha$-SMA immunostaining. (a) CCl , (b) FR50 mg/kg/day, and (c) FR100 mg/ $\mathrm{kg} /$ day $(\times 100)$. (d) Semiquantitative analyses of $\alpha$-SMA-stained areas showed a significant increase in $\alpha$-SMA-stained area following $\mathrm{CCl}_{4}$ treatment and significantly reduced the area in both FR50 and FR100 groups than in $\mathrm{CCl}_{4}$ and control (olive oil) rats. Results are expressed as mean \pm s.d. ${ }^{*} P<0.05$, $* * P<0.01$.

$P<0.01$ and $\mathrm{CCl}_{4}$ vs FR100; 64605.1 \pm 9070.9 vs $9749.8 \pm$ 5493.8; $P<0.01$ ) (Figure 2).

\section{Hepatic Hydroxyproline Content}

The hepatic hydroxyproline content of FR-treated rats was significantly lower when compared with the $\mathrm{CCl}_{4}$ group in a dose-dependent manner $\left(\mathrm{CCl}_{4}\right.$ vs $\quad$ FR50; $0.92 \pm 0.33$ vs $0.45 \pm 0.10 \mu \mathrm{mol} / 100 \mathrm{mg}$ liver; $P<0.05$ and $\mathrm{CCl}_{4}$ vs FR100; $0.92 \pm 0.33$ vs $0.32 \pm 0.06 \mu \mathrm{mol} / 100 \mathrm{mg}$ liver; $P<0.01$ ) (Figure 3 ).

\section{Activity of p38 MAPK}

Activation of p38 MAPK was assessed by Western blot analysis. A ratio of the phosphorylated and nonphosphorylated p38 MAPK was determined and was considered as the activity status of p38. In cirrhotic livers, p38 MAPK was significantly activated (olive oil vs $\mathrm{CCl}_{4} ; 0.54 \pm 0.37$ vs $1.25 \pm 0.59 ; P<0.05)$. Furthermore, FR inhibited activity of p38 MAPK $\left(\mathrm{CCl}_{4}\right.$ vs FR50; $1.25 \pm 0.59$ vs $0.63 \pm 0.18 ; P<0.05$ and $\mathrm{CCl}_{4}$ vs $\mathrm{FR} 100 ; 1.25 \pm 0.59$ vs $\left.0.50 \pm 0.13 ; P<0.05\right)$ (Figure 4).

\section{Expression of Smad4, TIMP-1, and Runx2}

Smad4 was strongly expressed in the nucleus and cytoplasm of spindle-shaped cells in periportal and perisinusoidal areas, and FR treatment significantly reduced Smad 4 expression in both these areas. The percentages of Smad4-positive cells were 30,12, and 9.2 in the $\mathrm{CCl}_{4}, \mathrm{FR} 50$, and FR100 group, respectively $\left(P<0.01, \mathrm{CCl}_{4}\right.$ vs FR50 and FR100) (Figure 5).

Similarly, a strong expression of TIMP-1 was noticed in the perisinusoidal and, occasionally, in the sinusoidal endothelial cells in the $\mathrm{CCl}_{4}$ livers. In contrast, only a weak and sparse TIMP-1 staining was detected in the periportal region in FR groups (Figure 6).

The mRNA expression of Runx2 in the livers was confirmed by RT-PCR analysis. Its expression in the cirrhotic liver was significantly high (olive oil vs $\mathrm{CCl}_{4} ; 0.59 \pm 0.13$ vs 


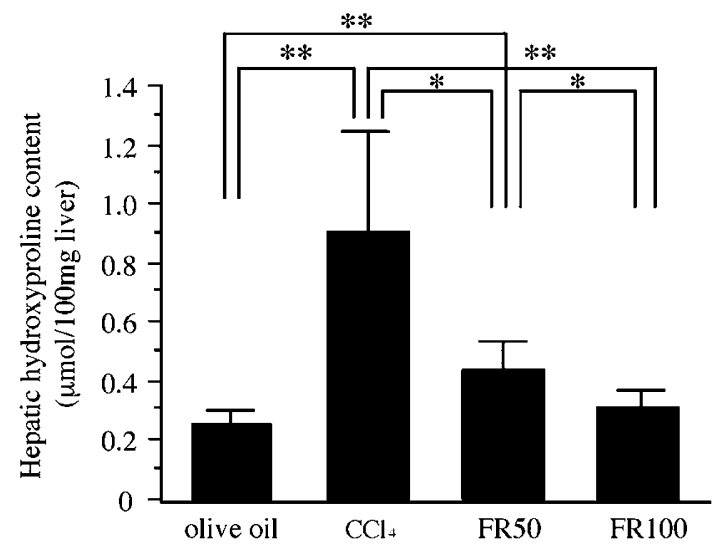

Figure 3 Hepatic hydroxyproline content. Hepatic hydroxyproline content as determined by using HPLC system shows a significant reduction in both FR50 and FR100 groups than in $\mathrm{CCl}_{4}$ rats, and a dose-dependent reduction in hydroxyproline content was noticed for FR treatment. Results are expressed as mean \pm s.d. ${ }^{*} P<0.05,{ }^{* *} P<0.01$.
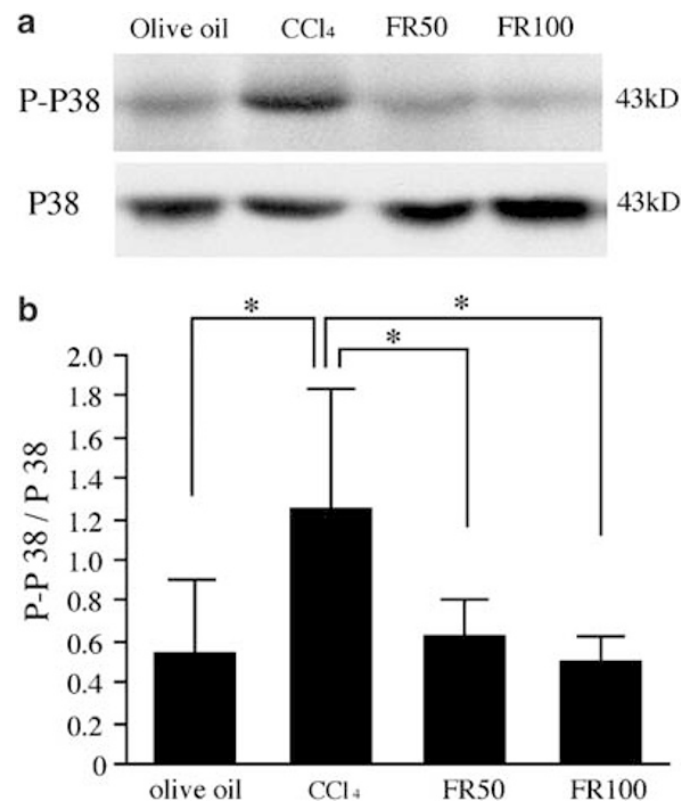

Figure 4 Effect of FR treatment on expression of p38 MAPK. (a) Western blot analysis of p38 MAPK and phosphorylated p38 MAPK. (b) Densitometric analysis of p38 MAPK expression is shown. Bars indicate the ratio of phosphorylated p38 MAPK/p38 MAPK. Results are expressed as mean \pm s.d. ${ }^{*} P<0.05,{ }^{*} P<0.01$.

$0.96 \pm 0.17 ; P<0.01)$. Moreover, FR significantly inhibited expression of Runx2 ( $\mathrm{CCl}_{4}$ vs FR50; $0.96 \pm 0.17$ vs $0.68 \pm 0.08$; $P<0.01$ and $\mathrm{CCl}_{4}$ vs FR100; $0.96 \pm 0.17$ vs $0.33 \pm 0.35$; $P<0.01$ ) (Figure 7).

\section{Discussion}

Liver cirrhosis is recognized as a leading cause of significant morbidity and mortality worldwide and also poses an enormous healthcare problem in Japan because of its high mortality rate. Certain percentages of these patients with cirrhosis succumb to hepatocellular carcinoma and thus further increase the mortality associated with cirrhosis. Treatment options for cirrhosis until now have been rather limited and have mostly relied on drugs that primarily restrict ongoing hepatic injury such as anti-viral therapies. Once the disease is in advanced stage, it remains difficult to treat successfully. To date, liver transplantation is the only hope for the treatment of end-stage liver cirrhosis with a 75\% 5 -year survival rate. ${ }^{21}$ However, an increasing demand of organs with restricted supply, issues of histocompatibility, and comorbid factors associated with cirrhosis mean that not every patient with cirrhosis is eligible for transplantation. Therefore, improved understanding of the underlying disease process and effective anti-fibrotic treatment regimens are needed urgently. Results of this study show that FR may fulfill this purpose and if proves effective in other studies, then FR would be a new therapeutic option for the prevention and treatment of cirrhosis in the clinic.

Initially, it was thought that HSCs located in the perisinusoidal area are the major type of cells responsible for hepatic fibrosis. However, several recent scholarly articles showed that several other hepatic resident mesenchymal cells with myofibroblast-like phenotype are also responsible for hepatic fibrosis. ${ }^{1-4}$ In this study, we found strong $\alpha$-SMA staining in both perisinusoidal and periportal areas, but hepatic fibrosis, as detected by azan staining, was predominantly noticed in the portal-central area and only a weak staining, in the perisinusoidal area. This may implicate that HSCs, which are primarily located in the perisinusoidal region, may not play a predominant role in hepatic fibrosis in this $\mathrm{CCl}_{4}$ induced cirrhosis model. FR treatment produced significant reduction in $\alpha$-SMA staining however, traces of both $\alpha$-SMA and azan staining could be noticed in the periportal and pericentral areas indicating that the myofibroblasts residing in the periportal and pericentral regions are relatively less sensitive to FR treatment, and a different regulatory mechanism for activation may exist in different kinds of myofibroblast residing in different region of the liver. Further studies with specific markers for each of these myofibroblasts are needed to reach a firm conclusion.

A number of novel anticirrhotic agents have been developed over the past few years including silibinin, HOEo77, safrinil, pentoxifiline IL-10, and herbal medicines such as Sho-kai-to and salvia miltiorrhiza. ${ }^{22}$ Interestingly, investigation into the mechanisms of actions of most of these drugs showed that blockade of the p38 MAPK pathway in the myofibroblasts was the key mechanism in amelioration of liver cirrhosis in these experiments. Given the central role of myofibroblasts in the development of liver cirrhosis, recently, Reeves et $\mathrm{l}^{23}$ showed a concrete evidence that the basal p38 MAPK activity of activated HSCs was higher than that of quiescent HSCs in an in vitro study, emphasizing the potential importance of p38 MAPK in activation of HSCs. In this study, we have extended the findings of the previous study by establishing that FR, a p38 specific inhibitor, abro- 

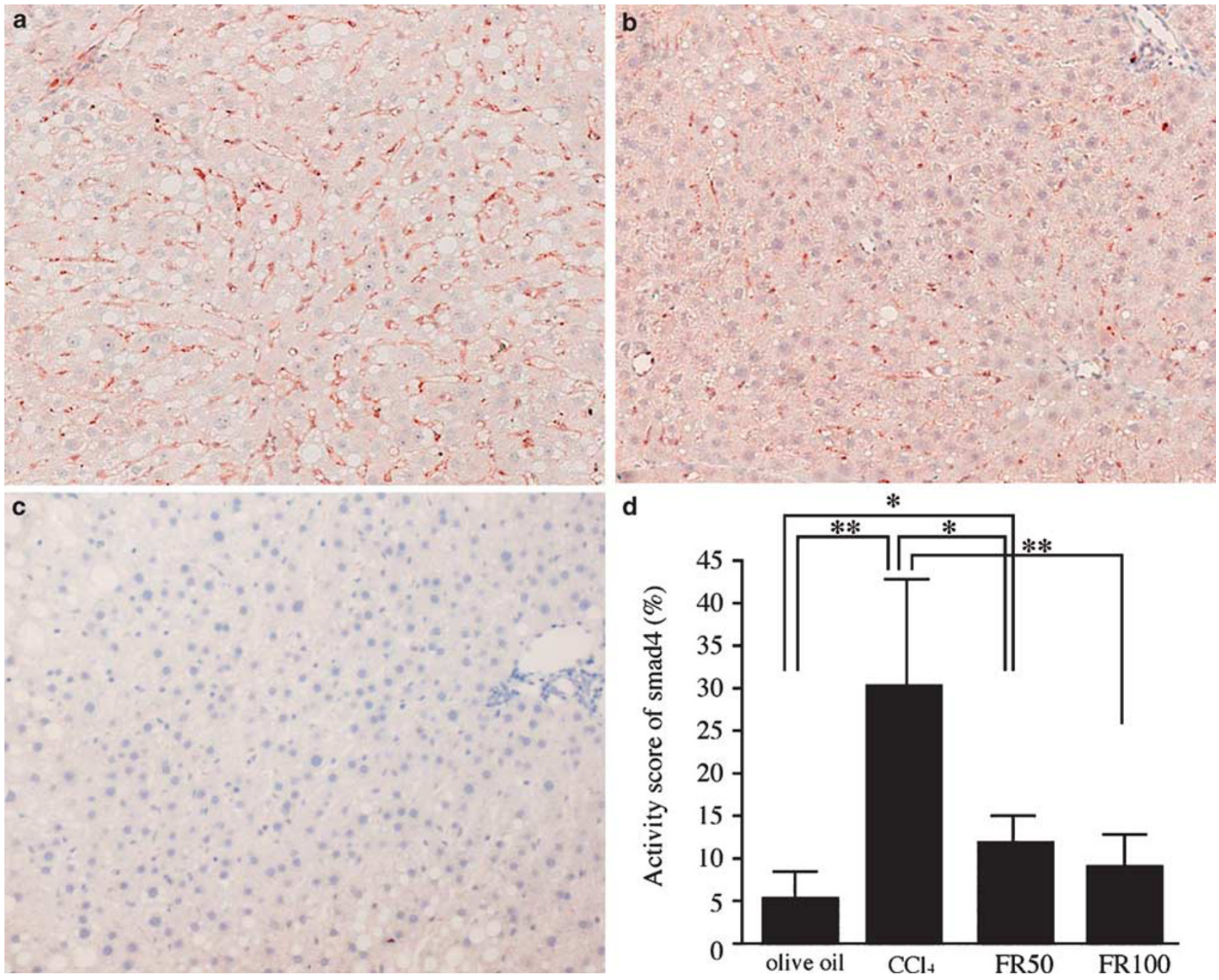

Figure 5 Representative liver sections showing immunohistochemical expression of Samd4. (a) $\mathrm{CCl}_{4}$, (b) $\mathrm{FR} 50 \mathrm{mg} / \mathrm{kg} / \mathrm{day}$, (c) FR 100 mg/kg/day ( $\times 100$ ). (d) Numbers of Samd4 positive nucleus in $\mathrm{FR}$ and $\mathrm{CCl}_{4}$ rats are shown. Nuclear immunoreactivity for Smad4 was described as the percentage of the number of positive myofibroblasts in one thousand myofibroblasts. Results are expressed as mean \pm s.d. ${ }^{*} P<0.05,{ }^{* *} P<0.01$.

gated the activation of myofibroblasts including HSCs and portal fibroblasts and, thus, ameliorated cirrhosis in rats. It was reported that the half-life of the collagen $\alpha-1$ is only $1.5 \mathrm{~h}$ in inactivated HSCs, whereas it is prolonged up to $24 \mathrm{~h}$ in activated HSCs. ${ }^{13}$ From this point of view, the once-a-day treatment regimen of FR, as used in this study, would be enough for effective inhibition of collagen synthesis in myofibroblasts because the half-life of FR is also approximately $24 \mathrm{~h}$. In a recent study, Matsuoka et $_{\text {al }}{ }^{18}$ have shown a dose-dependent inhibitory effect of FR in a bleomycin-induced pulumonary fibrosis model in rats. Similarly, in this study, FR treatment caused a significant reduction in azan and $\alpha$-SMA staining and also Smad4 expression in myofibroblasts.

The ameliorating effect of FR on cirrhosis could be attributed to its multiple actions including inhibition of cytokine activity, reduced signal transduction in myofibroblasts including HSCs, and portal fibroblasts, inhibition of che- motaxis of inflammatory cells into the liver and maintenance of ECM renewal by a fine tuning of the MMP/TIMP-1 balance. To date, an array of cytokines and growth factors, such as TGF- $\beta$, TNF- $\alpha$, bFGF, PDGF, myocyte enhancer factor 2 , and endothelin are thought to have a concerted action in the activation of myofibroblasts and an enhanced production of ECM including collagen $\alpha-1$. Among these different kinds of stimulating factors, TGF- $\beta$ plays a pivotal role in the activation of the myofibroblasts and cirrhosis formation. Much of the mechanisms by which TGF- $\beta$ enhances ECM production are emerging. Recent studies have elucidated that TGF- $\beta$ transduces the signal from its receptor to the nucleus not only via the classic Smad4 pathway but also via the Smad-dependent p38 MAPK pathway. ${ }^{24}$ An emerging body of evidence implicates that the intracellular p38 MAPK is an essential downstream component of the TGF- $\beta$ signaling pathway. Several in vitro studies indicate that TGF- $\beta$ when added in the culture medium activates the p38 pathway 

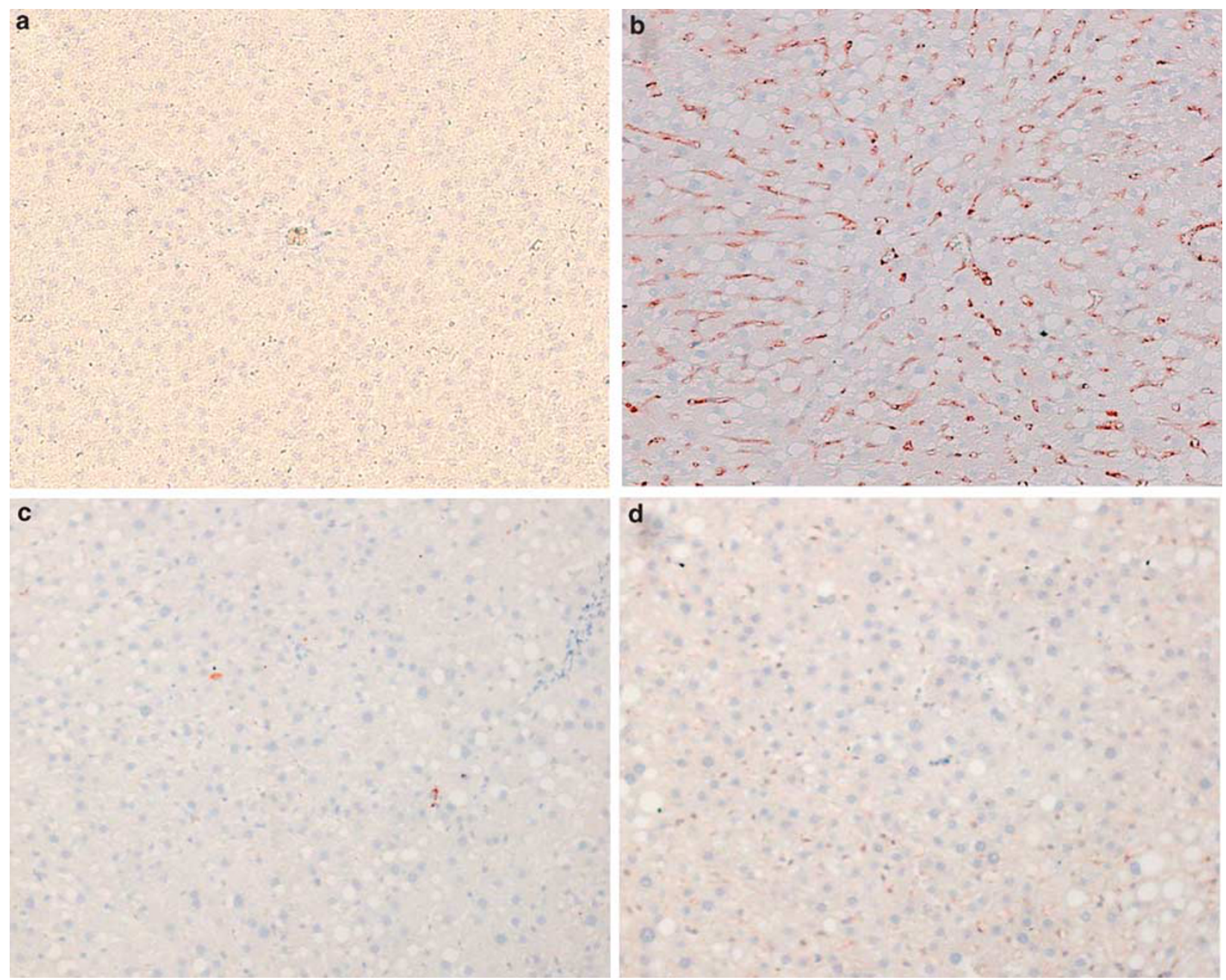

Figure 6 Immunohistochemical expression of TIMP-1 in representative liver sections. (a) Olive oil, (b) $\mathrm{CCl}_{4}$, (c) FR50 mg/kg/day, and (d) FR100 mg/kg/day $(\times 100)$.

within 15 min. ${ }^{25,26}$ TGF- $\beta$ activates TGF- $\beta$-activated kinase 1 (TAK1) and, in turn, TAK1 activates MKK3 and MKK6 both of which are activators of p38 MAPK. ${ }^{27,28}$ Phosphorylated p38 MAPK activates activating transcription factor 2 (ATF-2), and the transcription complex containing Smad4 and ATF-2 is responsible for the activation of the myofibroblasts. ${ }^{29}$ Very recently, Runx2 has been reported to be a new important downstream target of the TGF/Samd4 pathway, and an enhanced expression of Runx 2 has been noticed in activated HSCs in an in vitro study. ${ }^{30}$ Interestingly, it was reported that Smad4 does not induce Runx2 expression directly and an additional step of de novo protein synthesis is required. Subsequently, the authors identified p38 as the intermediate protein connecting Smad4 and Runx2 in a mouse pluripotent cell line $(\mathrm{C} 2 \mathrm{Cl} 2)$ and therefore, it indicated that a possible 'cross-talk' between the p38 and Runx2 pathways is existing. ${ }^{31}$ In osteognesis, Runx 2 controls directly the collagen $\alpha-1$ by binding the osteoblast-specific cis-acting elements (OSE2), ${ }^{15}$ and the phosphorylation of Runx2 by the MAPK pathway plays an important role in the activity of Runx2. ${ }^{16}$ Moreover, recently, it has been reported that $\mathrm{CCl}_{4}$ activates myfibroblasts by inducing galectin-3 expression, and this expression is regulated by Runx $2 .{ }^{32,33}$ In accordance with the results of previous studies, we also found a significant role of Runx2 in hepatic fibrosis, and Runx2 expression was significantly reduced by FR treatment. Further, in vitro studies involving hepatic myofibroblasts isolated from cirrhotic livers are necessary to prove the role of Runx2 in cirrhosis. P38 exerts its pro-fibrotic effect not only at the transcriptional level but also at the posttranscriptional level. It is well documented that p38 stabilizes mRNAs of profibrogenic agents such as TNF- $\alpha$ and cyclooxygenase- 2 and also stabilizes mRNA of collagen $\alpha-1 .^{34}$ Therefore, the protective effect of FR in this model could be partially attributable to enhanced degradation of mRNA of pro-fibrotic agents and collagen $\alpha-1$ itself.

Infiltration of liver by inflammatory cells is a predominant feature of cirrhosis in $\mathrm{CCl}_{4}$-treated rats, and FR significantly inhibited infiltration of these cells in the FR-treated rats. Similarly, Wada et $a l^{17}$ reported a marked inhibition of 

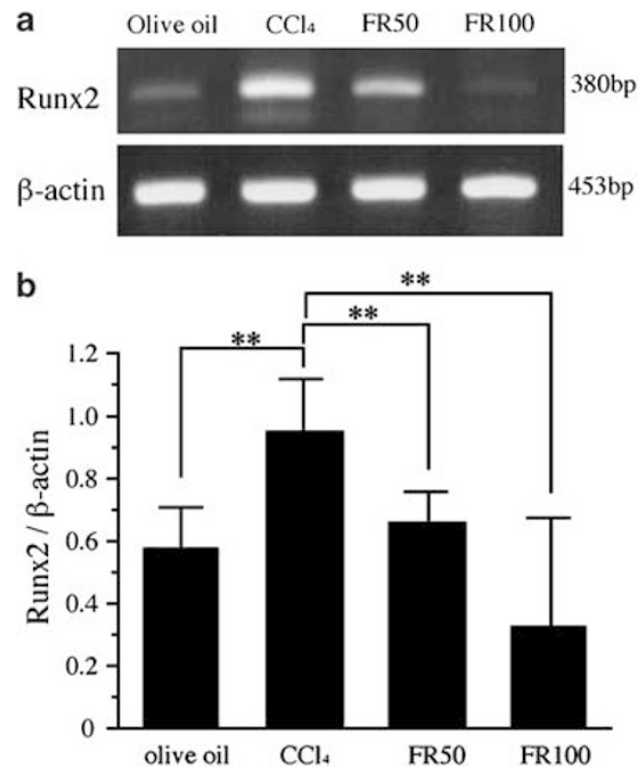

Figure 7 Effect of FR treatment on Runx2 mRNA expression. (a) RT-PCR of Runx2 mRNA in different groups. (b) Amounts of the Runx2 mRNA as determined by densitometric analysis are shown. The samples were evaluated in comparison with the PCR product to $\beta$-actin. Results are expressed as mean \pm s.d. ${ }^{\star} P<0.05,{ }^{*} P<0.01$.

macrophage infiltration by FR treatment in a model of crescentic glomerulonephritis in rats. Very recently, Yao and $\mathrm{Yue}^{20}$ have reported ameliorating effect of FR in an acute liver injury model induced by BCG and LPS in rats. In their study, FR significantly inhibited TNF- $\alpha$ and IL-1 release from the activated Kupffer cells. Kitamura et $a l^{35}$ revealed that TNF- $\alpha$ released from Kupffer cells activated HSCs and induced hepatic fibrosis. Therefore, it seems that FR treatment might have produced salutary effect on liver cirrhosis, at least partially, by inhibiting infiltration of inflammatory cells into the cirrhotic liver, as well as by inhibition of release of inflammatory cytokines.

In general, TGF- $\beta$ stimulates expression of TIMP- 1 via the p38 MAPK pathway. ${ }^{25}$ Recently, it has been noticed that Runx 2 induced by TGF- $\beta$ binds to the UTE- 1 sequence in the TIMP-1 promoter and regulates its transcription. ${ }^{30}$ In this study, we have shown a significant reduction in TIMP-1 expression in the FR-treated rats, and it might be possible that FR treatment caused an enhanced fibrolysis by inhibiting the TGF- $\beta /$ p38/Runx2/TIMP- 1 axis in myofibroblasts and may implicate that FR prevents liver cirrhosis not only by inhibiting fibrogenesis, but also by accelerating the fibrolysis.

Histological sections of FR-treated rats showed mild fatty changes in the FR-treated rats. However, the liver function was improved in FR-treated rats than in $\mathrm{CCl}_{4}$. This fatty change might be attributed to modulation of expression of the peroxisome proliferator-activated receptor $\gamma(\operatorname{PPAR} \gamma)$ by p38 MAPK in this $\mathrm{CCl}_{4}$ /olive oil-induced liver cirrhosis model. In adipocytes, inhibition of p38 MAPK reduces $\operatorname{PPAR} \gamma$ expression and prevents their differentiation. Also

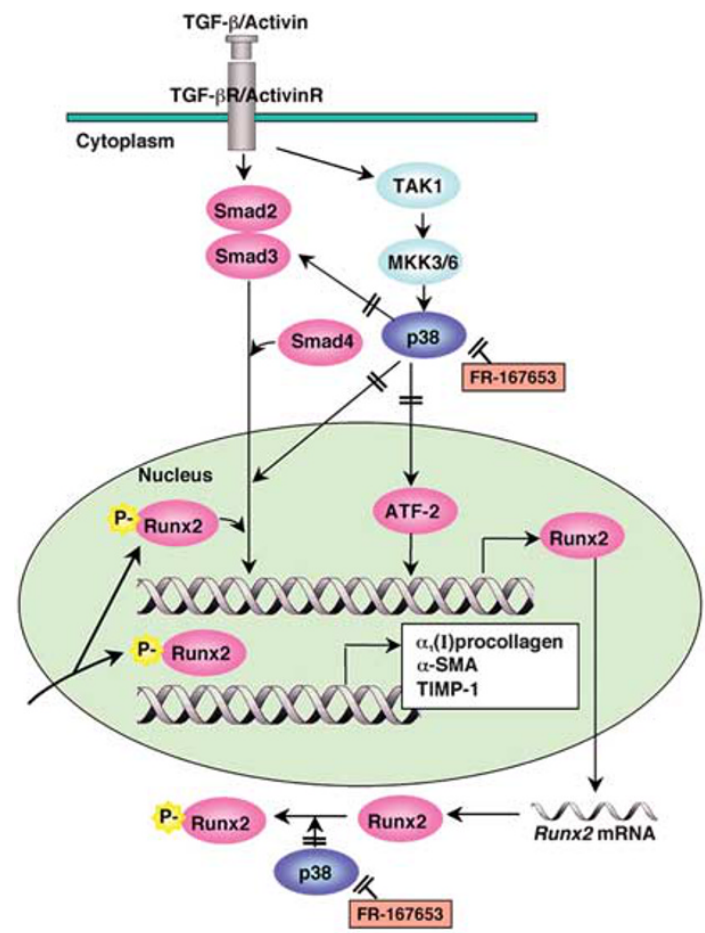

Figure 8 Schematic representation of signaling cascades in the myofibroblasts. TAK1, TGF- $\beta$-activated kinase 1 ; MKK, MAPK kinase; ATF-2, activating transcription factor-2; TIMP-1, tissue inhibitor of metalloproteinase-1.

$\mathrm{CCl}_{4}$ induces fatty degeneration and necrosis by its hepatotoxicity, and then fibrosis progressively develops around these injured areas. In addition, $\mathrm{CCl}_{4}$ decreases release of lipoprotein from the liver to the serum and induces fatty degeneration. In this cirrhotic model, FR treatment produced a low lipid turnover in liver by both inhibition of PPAR $\gamma$ and $\mathrm{CCl}_{4}$-induced liver injury. In fact, in the preliminary research, we evaluated the effect of FR in a bile duct ligation model of liver cirrhosis in rats and FR had an ameliorating effect without any significant fatty changes (data not shown). Very recently, it has been reported that $\operatorname{PPAR} \gamma$ ligand prevents the activation of HSCs and hepatic steatosis. ${ }^{36,37}$ Therefore, it would be intriguing to find a synergistic effect between a PPAR $\gamma$ ligand and FR in a liver cirrhotic model.

It has been reported that FR attenuates hepatic ischemiareperfusion injury and promotes liver regeneration after hepatectomy. ${ }^{20}$ To perform an extended liver resection in patients with hepatocellular carcinoma associated with advanced cirrhosis remains a challenge for the surgeons. Perioperative administration of FR may improve cirrhosis, attenuate reperfusion injury and accelerate regeneration and, thus may permit a safe liver resection even in patients with advanced cirrhosis. However, further studies are necessary before its use in the clinic.

Taken together, results of this study indicate that FR may have a salutary effect on $\mathrm{CCl}_{4}$-induced liver cirrhosis in rats. This effect might be due to direct inhibition of the p38 
MAPK pathway in hepatic myofibroblasts including HSCs and portal myofibroblats. The antifibrogenic effect of FR is also partially attributable to downregulation of Samd4, Runx2, and TIMP1 pathways in cirrhotic liver (Figure 8). FR may prove valuable in designing anti-fibrotic therapy and further studies should be performed before use of this drug in the clinic.

\section{ACKNOWLEDGEMENT}

We are grateful to Mrs Miki Asazu and Mika Nada for their excellent technical assistance. We also thank Fujisawa Pharmaceutical Co. Ltd (Osaka, Japan) for kindly providing the FR- 167653 .

1. Guyot $C$, Lepreux $S$, Combe $C$, et al. Hepatic fibrosis and cirrhosis: the (myo)fibroblastic cell subpopulations involved. Int J Biochem Cell Biol 2006;38:135-151.

2. Ramadori G, Saile B. Portal tract fibrogenesis in the liver. Lab Invest 2004;84:153-159.

3. Kinnman N, Francoz C, Barbu V, et al. The myofibroblastic conversion of peribiliary fibrogenic cells distinct from hepatic stellate cells is stimulated by platelet-derived growth factor during liver fibrogenesis. Lab Invest 2003;83:163-173.

4. Tuchweber B, Desmouliere A, Bochaton-Piallat ML, et al. Proliferation and phenotypic modulation of portal fibroblasts in the early stages of cholestatic fibrosis in the rat. Lab Invest 1996;74:265-278.

5. Bissell DM, Roulot D, George J. Transforming growth factor beta and the liver. Hepatology 2001;34:859-867.

6. Sugiyama M, Ichida $T$, Sato $T$, et al. Expression of activin A is increased in cirrhotic and fibrotic rat livers. Gastroenterology 1998;114:550-558.

7. Cho JJ, Hocher B, Herbst $\mathrm{H}$, et al. An oral endothelin-A receptor antagonist blocks collagen synthesis and deposition in advanced rat liver fibrosis. Gastroenterology 2000;118:1169-1178.

8. Kinnman N, Goria O, Wendum D, et al. Hepatic stellate cell proliferation is an early platelet-derived growth factor-mediated cellular event in rat cholestatic liver injury. Lab Invest 2001;81:1709-1716.

9. Komuves $L G$, Feren $A$, Jones $A L$, et al. Expression of epidermal growth factor and its receptor in cirrhotic liver disease. J Histochem Cytochem 2000;48:821-830.

10. Sudo $\mathrm{K}$, Yamada $\mathrm{Y}$, Moriwaki $\mathrm{H}$, et al. Lack of tumor necrosis factor receptor type 1 inhibits liver fibrosis induced by carbon tetrachloride in mice. Cytokine 2005;29:236-244.

11. Parsons CJ, Bradford BU, Pan CQ, et al. Antifibrotic effects of a tissue inhibitor of metalloproteinase-1 antibody on established liver fibrosis in rats. Hepatology 2004;40:1106-1115.

12. Chang L, Karin M. Mammalian MAP kinase signalling cascades. Nature 2001;410:37-40.

13. Tsukada S, Westwick JK, Ikejima K, et al. SMAD and p38 MAPK signaling pathways independently regulate alpha1(I) collagen gene expression in unstimulated and transforming growth factor-beta-stimulated hepatic stellate cells. J Biol Chem 2005;280:10055-10064.

14. Ito Y. Oncogenic potential of the RUNX gene family: 'overview'. Oncogene 2004;23:4198-4208.

15. Ducy $\mathrm{P}$, Zhang R, Geoffroy $\mathrm{V}$, et al. Osf2/Cbfa1: a transcriptional activator of osteoblast differentiation. Cell 1997;89:747-754.

16. Xiao $G$, Jiang $D$, Thomas $P$, et al. MAPK pathways activate and phosphorylate the osteoblast-specific transcription factor, Cbfa1. J Biol Chem 2000;275:4453-4459.

17. Wada T, Furuichi K, Sakai N, et al. A new anti-inflammatory compound, FR167653, ameliorates crescentic glomerulonephritis in Wistar-Kyoto rats. J Am Soc Nephrol 2000;11:1534-1541.
18. Matsuoka H, Arai T, Mori M, et al. A p38 MAPK inhibitor, FR-167653, ameliorates murine bleomycin-induced pulmonary fibrosis. Am J Physiol Lung Cell Mol Physiol 2002;283:L103-L112.

19. Tsutsumi R, Kamohara Y, Eguchi S, et al. Selective suppression of initial cytokine response facilitates liver regeneration after extensive hepatectomy in rats. Hepatogastroenterology 2004;51:701-704.

20. Yao HW, Yue L. Effect and mechanisms of FR167653, a dual inhibitor of TNF-alpha and IL-1, on BCG plus LPS induced-liver injury. Inflamm Res 2005;54:471-477.

21. Selberg O, Bottcher J, Tusch G, et al. Identification of high- and low-risk patients before liver transplantation: a prospective cohort study of nutritional and metabolic parameters in 150 patients. Hepatology 1997;25:652-657.

22. Burt AD. Pathobiology of hepatic stellate cells. J Gastroenterol 1999;34:299-304.

23. Reeves $\mathrm{HL}$, Dack $\mathrm{CL}$, Peak $\mathrm{M}$, et al. Stress-activated protein kinases in the activation of rat hepatic stellate cells in culture. J Hepatol 2000;32:465-472.

24. Derynck R, Zhang YE. Smad-dependent and Smad-independent pathways in TGF-beta family signalling. Nature 2003;425:577-584.

25. Cao Q, Mak KM, Lieber CS. DLPC decreases TGF-beta1-induced collagen mRNA by inhibiting p38 MAPK in hepatic stellate cells. Am J Physiol Gastrointest Liver Physiol 2002;283:G1051-G1061.

26. Varela-Rey M, Montiel-Duarte C, Oses-Prieto JA, et al. p38 MAPK mediates the regulation of alpha1(I) procollagen mRNA levels by TNF-alpha and TGF-beta in a cell line of rat hepatic stellate cells(1). FEBS Lett 2002;528:133-138.

27. Yamaguchi K, Shirakabe K, Shibuya H, et al. Identification of a member of the MAPKKK family as a potential mediator of TGF-beta signal transduction. Science 1995;270:2008-2011.

28. Schnabl B, Bradham CA, Bennett BL, et al. TAK1/JNK and p38 have opposite effects on rat hepatic stellate cells. Hepatology 2001;34: 953-963.

29. Hanafusa $\mathrm{H}$, Ninomiya-Tsuji J, Masuyama $\mathrm{N}$, et al. Involvement of the p38 mitogen-activated protein kinase pathway in transforming growth factor-beta-induced gene expression. J Biol Chem 1999;274:2716127167.

30. Bertrand-Philippe M, Ruddell RG, Arthur MJ, et al. Regulation of tissue inhibitor of metalloproteinase 1 gene transcription by RUNX1 and RUNX2. J Biol Chem 2004;279:24530-24539.

31. Lee KS, Hong SH, Bae SC. Both the Smad and p38 MAPK pathways play a crucial role in Runx2 expression following induction by transforming growth factor-beta and bone morphogenetic protein. Oncogene 2002;21:7156-7163.

32. Henderson NC, Mackinnon AC, Farnworth SL, et al. Galectin-3 regulates myofibroblast activation and hepatic fibrosis. Proc Natl Acad Sci USA 2006;103:5060-5065.

33. Stock M, Schafer H, Stricker S, et al. Expression of galectin-3 in skeletal tissues is controlled by Runx2. J Biol Chem 2003;278:17360-17367.

34. Dean JL, Sarsfield SJ, Tsounakou E, et al. p38 Mitogen-activated protein kinase stabilizes mRNAs that contain cyclooxygenase- 2 and tumor necrosis factor AU-rich elements by inhibiting deadenylation. J Biol Chem 2003;278:39470-39476.

35. Kitamura K, Nakamoto $\mathrm{Y}$, Akiyama M, et al. Pathogenic roles of tumor necrosis factor receptor p55-mediated signals in dimethylnitrosamineinduced murine liver fibrosis. Lab Invest 2002;82:571-583.

36. Marra F, Efsen E, Romanelli RG, et al. Ligands of peroxisome proliferator-activated receptor gamma modulate profibrogenic and proinflammatory actions in hepatic stellate cells. Gastroenterology 2000;119:466-478.

37. Kawaguchi K, Sakaida I, Tsuchiya M, et al. Pioglitazone prevents hepatic steatosis, fibrosis, and enzyme-altered lesions in rat liver cirrhosis induced by a choline-deficient L-amino acid-defined diet. Biochem Biophys Res Commun 2004;315:187-195. 